\title{
On the extraction of spectral quantities with open boundary conditions
}

\author{
Mattia Bruno*a, Piotr Korcyl ${ }^{a}$, Tomasz Korzec ${ }^{b}$, Stefano Lottini ${ }^{a}$ and Stefan \\ Schaefer $^{a}$ \\ a John von Neumann Institute for Computing (NIC), DESY \\ Platanenallee 6, D-15738 Zeuthen, Germany \\ ${ }^{b}$ Institut für Physik, Humboldt Universität zu Berlin \\ Newtonstr. 15, D-12489 Berlin, Germany
}

\begin{abstract}
We discuss methods to extract decay constants, meson masses and gluonic observables in the presence of open boundary conditions. The ensembles have been generated by the CLS effort and have $2+1$ flavors of $\mathrm{O}$ (a)-improved Wilson fermions with a small twisted-mass term as proposed by Lüscher and Palombi. We analyse the effect of the associated reweighting factors on the computation of different observables.
\end{abstract}

DESY $14-211$

$\mathrm{HU}-\mathrm{EP}-14 / 50$

The 32nd International Symposium on Lattice Field Theory,

23-28 June, 2014

Columbia University New York, NY

${ }^{*}$ Speaker. 


\section{Introduction}

To overcome the topological freezing which makes simulations at lattice spacings below $0.05 \mathrm{fm}$ practically impossible, open boundary conditions in the temporal direction have been proposed [1]. In our study we analyse the ensembles generated by the CLS effort with $2+1 \mathrm{O}(a)$-improved Wilson fermions and twisted-mass reweighting à la Lüscher-Palombi [2]. For the details of the algorithmic and physical parameters we refer to Refs. [3, 4]. In these Proceedings we discuss the consequences of the open boundaries in gluonic and fermionic observables, such as the scale parameter $t_{0}$ [5] and the pion mass and decay constant. In the last section we also examine the implications of the twisted-mass reweighting and its impact on the final results.

\section{Definitions}

The interpretation of the boundary state can be taken from Ref. [6], where Schrödingerfunctional boundary conditions are adopted. Our case is similar, since far away from the boundary the expectation value of an observable $O\left(x_{0}, \mathbf{x}\right)$ corresponds to the vacuum expectation value up to exponentially suppressed contributions from states with vacuum quantum numbers (e.g. a $2 \pi$ state in QCD or a scalar glueball in pure Yang-Mills theory). The gluonic observable under study is the energy density (clover-type discretisation of $\hat{G}_{\mu \nu}$ ) obtained from the Wilson flow at positive flow time [5]

$$
t^{2}\left\langle E\left(t, x_{0}\right)\right\rangle=t^{2} \frac{1}{L^{3}} \sum_{\mathbf{x}} \frac{1}{4}\left\langle\hat{G}_{\mu v}^{a}\left(x_{0}, \mathbf{x}\right) \hat{G}_{\mu v}^{a}\left(x_{0}, \mathbf{x}\right)\right\rangle,
$$

which we also use to compute the scale $t_{0}$, defined by the time $t$ at which the r.h.s of eq. (2.1) is equal to 0.3 . From the fermionic side, we measure the following two-point correlation functions stochastically $^{1}$ (throughout the paper $T$ and $L$ refer to the temporal and spatial extent of the lattice)

$$
f_{X}\left(x_{0}, y_{0}\right)=-\frac{a^{6}}{L^{3}} \sum_{\mathbf{x}, \mathbf{y}}\left\langle X^{r s}\left(x_{0}, \mathbf{x}\right) P^{s r}\left(y_{0}, \mathbf{y}\right)\right\rangle, \quad P^{r s}\left(y_{0}, \mathbf{y}\right)=\bar{\psi}^{r}(y) \gamma_{5} \psi^{s}(y),
$$

with $X^{r s}$ either $P^{r s}$ or $A_{0}^{r s}\left(y_{0}, \mathbf{y}\right)=\bar{\psi}^{r}(y) \gamma_{0} \gamma_{5} \psi^{s}(y)$ and $r, s$ flavor indices. We compute the effective mass using

$$
a m_{\mathrm{eff}}\left(x_{0}+a / 2, y_{0}\right)=\log \frac{f_{\mathrm{P}}\left(x_{0}, y_{0}\right)}{f_{\mathrm{P}}\left(x_{0}+a, y_{0}\right)}, \quad m_{\mathrm{eff}}^{\mathrm{aver}}\left(y_{0}\right)=\frac{1}{N_{\text {points }}} \sum_{x_{0} \in \text { plateau }} m_{\mathrm{eff}}\left(x_{0}, y_{0}\right) .
$$

\section{Cutoff effects}

In Figure 1 we show the profile of the energy density and the effective pion mass as the boundaries at $x_{0}=0$ and $x_{0}=T$ are respectively approached. In $E\left(t_{0}, x_{0}\right)$ the effects seem to be dominantly of $\mathrm{O}(a)$, indeed the plateau starts (for our three lattice spacings) always in the same region of $x_{0} / a$. Moreover effects from the sea-quark masses are not visible, even at the boundaries, given the sub-percent precision of this observable. In the pion mass, sea-quark effects close to the boundaries are present, though much smaller than the dependence on the spacing.

\footnotetext{
${ }^{1}$ The measurement code is publicly available at https://github.com/to-ko/mesons.
} 

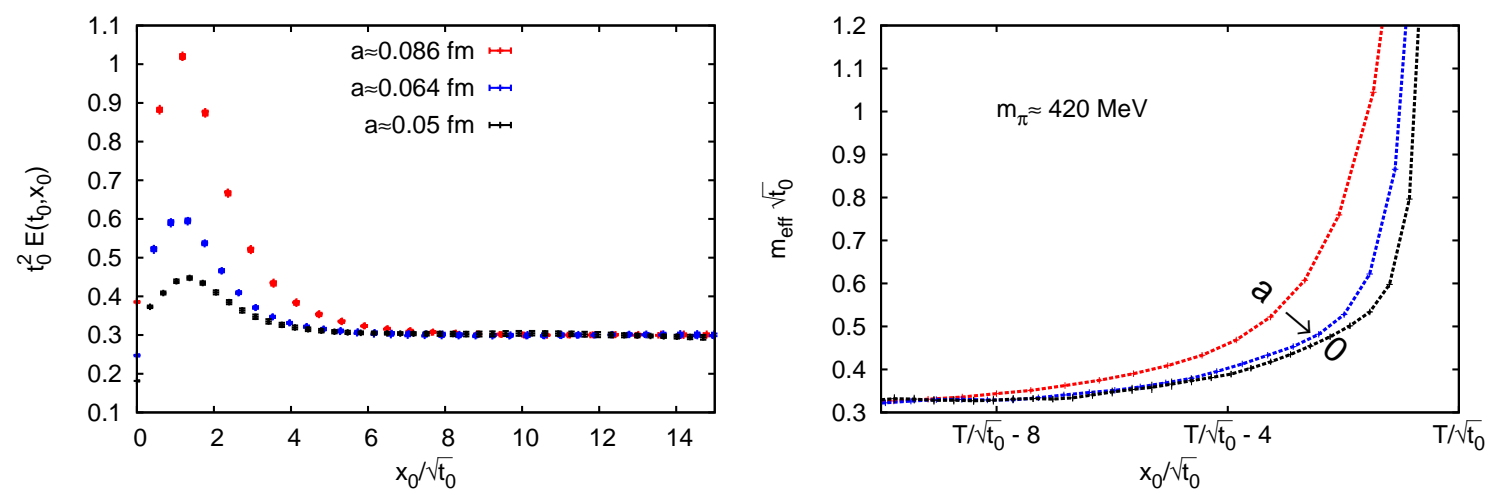

Figure 1: Left: cutoff effects of the energy density in units of $t_{0}$ close to the boundary at $x_{0}=0$ for three lattice spacings and pion masses from $200 \mathrm{MeV}$ to $420 \mathrm{MeV}$. Right: cutoff effects of $m_{\mathrm{eff}}\left(x_{0}+a / 2, y_{0}=a\right)$ close to the boundary at $x_{0}=T$ in units of $\sqrt{t_{0}}$.

As a measure of the goodness of our plateaux we fit both quantities to a constant and we compare the uncorrelated $\chi^{2}$ to $\chi_{\exp }^{2}$, the expected value computed from the measured covariance matrix ${ }^{2}$, as a function of the distance from the boundary. We find that in the central part of the lattice $\chi^{2} / \chi_{\exp }^{2}$ is around one. For the energy density we take the first time slice where $\chi^{2} / \chi_{\exp }^{2} \approx 1$ as the beginning of the plateau, while for the effective mass eq. (2.3) we consider an additional distance of $0.25 \mathrm{fm}$ to avoid residual excited states (for a multi-state analysis see Ref. [4]).

\section{Decay constants}

As a first attempt, one would naively place source and sink of the two-point functions in the middle of the lattice, to avoid boundary contaminations. However the additional contributions of the states excited by the source operator would reduce even more the portion of the lattice usable for the extraction of a mass or a decay constant. For this reason we measure the correlators with the source close to one boundary and the sink in the bulk. When possible we always make use of time reversal symmetry by averaging correlation functions at $\left(x_{0}, y_{0}\right)$ and $\left(T-x_{0}, T-y_{0}\right)$.

Now, the expectation values in eq. (2.2) take the form (for sufficiently large $x_{0}-y_{0}$ and $T-x_{0}$ )

$$
f_{\mathrm{A}}\left(x_{0}, y_{0}\right)=A\left(y_{0}\right) \hat{f}_{\pi} e^{-m_{\text {eff }}\left(x_{0}-y_{0}\right)}, \quad f_{\mathrm{P}}\left(T-y_{0}, y_{0}\right)=A\left(y_{0}\right)^{2} e^{-m_{\text {eff }}\left(T-2 y_{0}\right)} .
$$

Note that $\hat{f}_{\pi}$ indicates the matrix element $\left\langle 0\left|\mathbb{A}_{0}\right| \pi\right\rangle$ related to the pion decay constant through an additional normalisation factor. $A\left(y_{0}\right)$ is the amplitude related to the matrix element $\langle 0|\mathbb{P}| \pi\rangle$ which depends on the distance from the boundary $y_{0}$ because we place $y_{0}$ close to one boundary where the excited states from the boundary are not yet exponentially suppressed.

In Ref. [6], where the same type of observables was studied in the Schrödinger functional setup, the following ratio to compute the pion decay constant has been proposed

$$
F_{\pi}^{\text {bare }} \propto \frac{f_{\mathrm{A}}\left(x_{0}, y_{0}\right)}{\sqrt{f_{\mathrm{P}}\left(T-y_{0}, y_{0}\right)}} e^{m_{\mathrm{eff}}\left(x_{0}-T / 2\right)} .
$$

\footnotetext{
${ }^{2}$ This method avoids the inversion of the covariance matrix.
} 

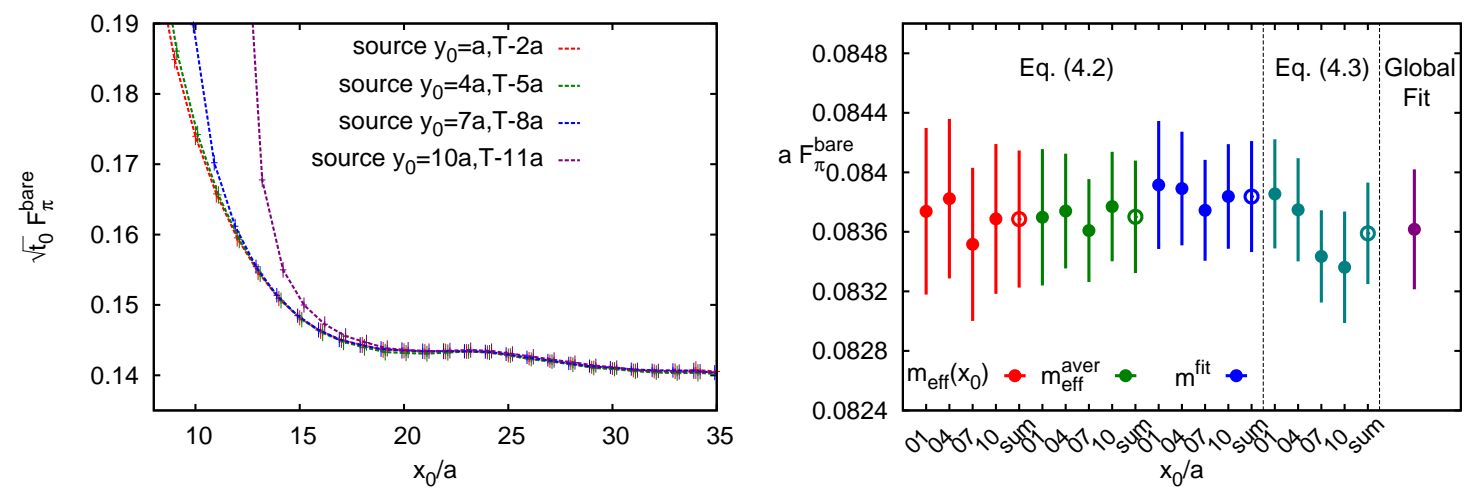

Figure 2: The left panel shows the pion decay constant computed from eq. (4.3) for several source positions. In the right panel we plot the results from eq. (4.2) using different definitions of $m_{\mathrm{eff}}$ (red and green points correspond to eq. (2.3), while blue points to the result of the global fit), from eq. (4.3) and from the global fit. The filled symbols are the results from correlators with source fixed to a certain time slice, while the open symbols correspond to the average over displaced sources. These tests have been done on an ensemble with $m_{\mathrm{K}}=m_{\pi} \approx 420 \mathrm{MeV}, a \approx 0.086 \mathrm{fm}$ and $8000 \mathrm{MDU}$.

Alternatively we consider here a new combination of two-point functions which cancels at the same time the amplitude $A\left(y_{0}\right)$ and the remaining exponential factor

$$
F_{\pi}^{\mathrm{bare}}\left(x_{0}, y_{0}\right) \propto \sqrt{\frac{f_{\mathrm{A}}\left(x_{0}, y_{0}\right) f_{\mathrm{A}}\left(x_{0}, T-y_{0}\right)}{f_{\mathrm{P}}\left(T-y_{0}, y_{0}\right)}} .
$$

In both cases a vacuum average in the plateau region $0 \ll x_{0} \ll T$ can be taken.

In principle the same average could be taken also for $y_{0}$, the position of the source. However as can be seen from Figure 2 (left panel), where we plot $F_{\pi}^{\text {bare }}$ obtained from eq. (4.3) for several $y_{0}$, the correlation functions computed from displaced sources are fully correlated in the bulk. Therefore, there is no advantage in averaging over several $y_{0}$. In Figure 2 (right panel) we plot the results of $F_{\pi}^{\text {bare }}$ (after the plateau average) computed with different approaches. All the methods investigated here return results in agreement with each other and with the same precision. Since the global fit is not more precise than the other two methods and the one proposed in Ref. [6] is sensitive to the choice of the effective mass used in the exponential factor of eq. (4.2), we prefer to use eq. (4.3) because it does not suffer from the ambiguity of the choice of $m_{\mathrm{eff}}$ and it turns out to be much easier to implement.

\section{Impact of reweighting on observables}

Simulations with a small pion mass are not only expensive in terms of computational cost but can also run into instabilities if accidental zero-modes of the Wilson Dirac operator occur. However such a problem can be cured by regularizing the fermion determinant with a small twisted-mass term [2]. Our set of ensembles has been generated with the so-called type II twisted-mass reweighting (applied only to the Schur complement $\hat{Q}$ of the asymmetric even-odd preconditioning [7]), 

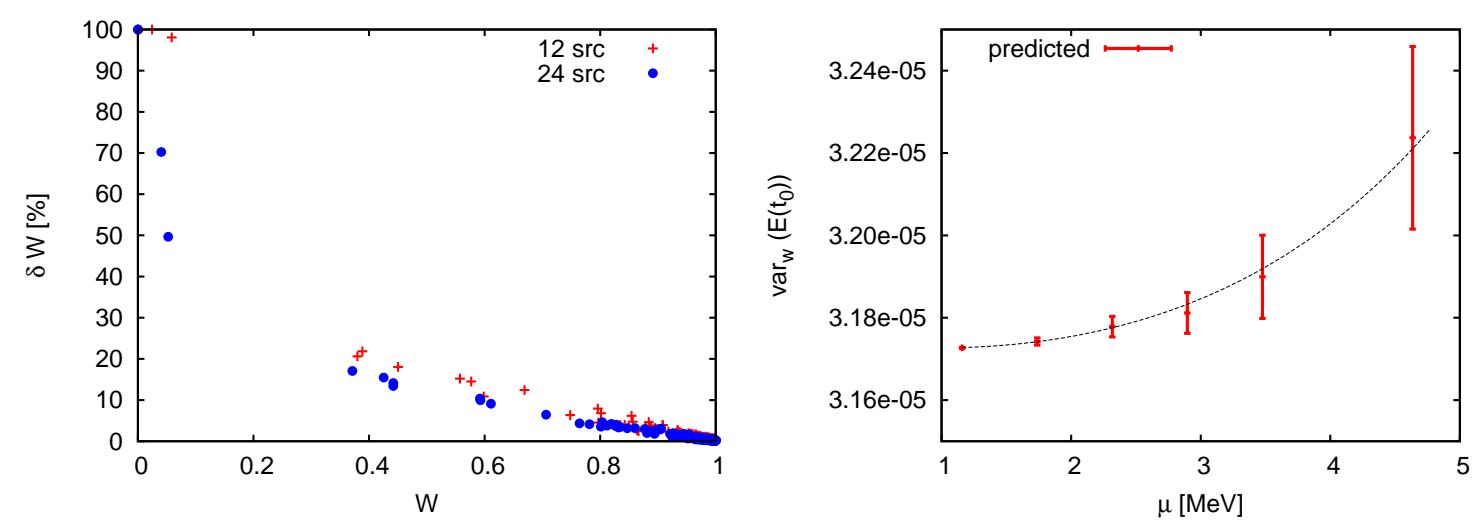

Figure 3: Left: Error of the reweighting factor as a function of its central value configuration by configuration for an ensemble with $m_{\pi} \approx 280 \mathrm{MeV}, a \approx 0.086 \mathrm{fm}, 8000 \mathrm{MDU}$ and $\mu=10^{-3}$. Increasing the number of stochastic sources improves the precision only if $W \gtrsim 0.5$. Right: the dotted line is drawn only to guide the eye. The points are obtained by using the reweighting factor of eq. (5.2) in eq. (5.4) with increasing $\mu$, computed always on the same ensemble (generated with $\mu=0.5 \cdot 10^{-3}, a \approx 0.086 \mathrm{fm}, m_{\pi} \approx 280 \mathrm{MeV}$ ).

whose fluctuations are smaller in the UV-regime

$$
S_{\mathrm{f}}=-\log \operatorname{det} \frac{\left(\hat{Q}^{2}+\mu^{2}\right)^{2}}{\hat{Q}^{2}+2 \mu^{2}}-2 \log \operatorname{det} Q_{\mathrm{oo}}, \quad \hat{Q}=\gamma_{5} \hat{D},
$$

which requires the computation of the corresponding reweighting factor $W$ to obtain the observables in the underlying theory $\left(\mu=0, S_{\mathrm{f}}=-\log \operatorname{det} \hat{Q}^{2}-2 \log \operatorname{det} Q_{\mathrm{oo}}\right)$

$$
W=\operatorname{det} \frac{\hat{Q}^{2}\left(\hat{Q}^{2}+2 \mu^{2}\right)}{\left(\hat{Q}^{2}+\mu\right)^{2}} .
$$

The reweighting factors are estimated stochastically, therefore, at first place, it is important to find an optimal number of stochastic sources which balances between computational cost and error size, but also to check the convergence of the variance. Indeed, as can be seen from Figure 3 (left panel), the relative error of the reweighting factor grows as the mean value of $W$ goes to zero. In general when $W$ is below 0.8 the stochastic error is larger than $10 \%$ and starts to become relevant, but when $W$ is relatively small, say below 0.5 , the computation of the reweighting factor becomes problematic and even wrong for few gauge-field configurations [8]. Using a factorization of the determinant in eq. (5.2) à la Hasenbusch $[9,10]$ might help in these cases.

A second issue which we investigate here is the possibility that the fluctuations of the reweighting factors with the gauge-field configurations influence the precision of the reweighted observables. Clearly these fluctuations are controlled by the choice of $\mu$, for a fixed light quark mass. In general $\mu>0$ makes the regions of field space with near-zero modes of the Dirac operator accessible, but large values of $\mu$ tend to enhance this effect too much. For these configurations $W$ is close to zero while fermionic observables, such as meson correlation functions, develop "spikes" and during the reweighting procedure cancellations take place. Therefore if from one side the twisted mass improves the ergodicity of the algorithm, from the other it is a delicate parameter, because it controls the occurence of configurations with almost-zero fermionic determinant, for which the reweighting procedure might fail (especially without the determinant factorization). 

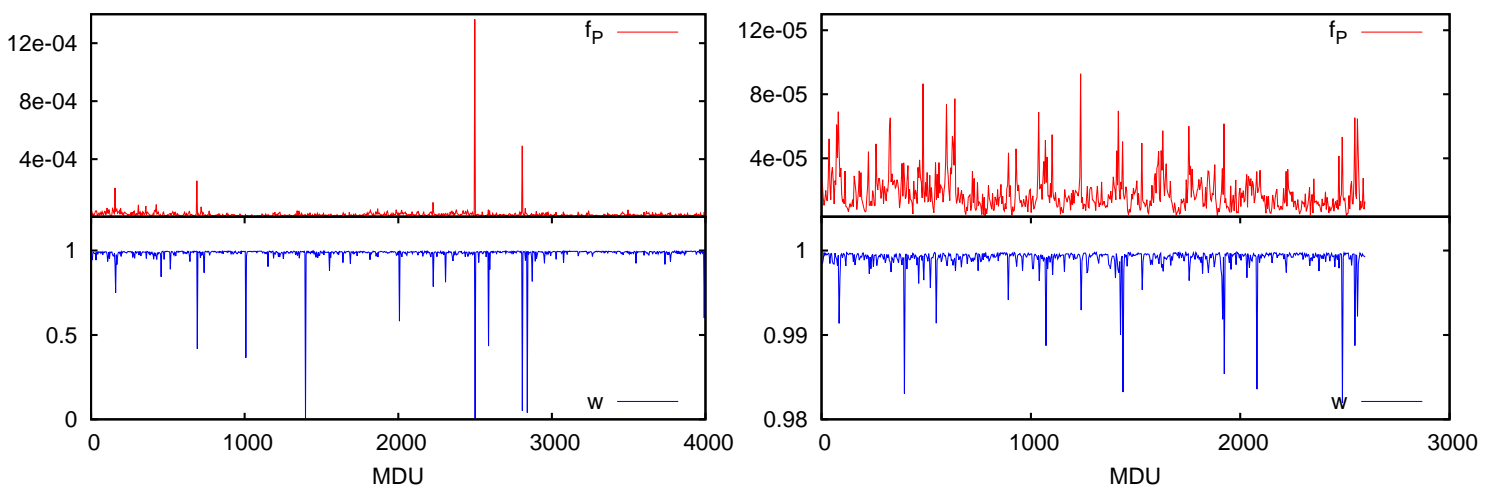

Figure 4: The red curves represent the Monte Carlo history of $f_{\mathrm{P}}\left(x_{0}, y_{0}\right)$ with $x_{0}=T / 2, y_{0}=a$, the blue curves show the fluctuations of the reweighting factor from eq. (5.2). Both lattices are $96 \times 32^{3}$ and have $m_{\pi} \approx 280 \mathrm{MeV}, a \approx 0.086 \mathrm{fm}$. Left: ensemble generated with $\mu=10^{-3}$. Right: ensemble generated with $\mu=0.5 \cdot 10^{-3}$. Note the difference in the scales of the vertical axes between left and right.

In Figure 4 we present the Monte Carlo histories of the twisted-mass reweighting factor and $f_{\mathrm{P}}\left(x_{0}, y_{0}\right)$ for two simulations which differ only by the choice of $\mu$. The fluctuations of both the observable and $W$ are suppressed by a factor 10 when $\mu \rightarrow \mu / 2$ (left $\rightarrow$ right), for this particular setup. This example illustrates that $\mu$ must be chosen with care.

Since a priori an optimal choice of $\mu$ is unclear, especially in pioneering simulations like ours, it is necessary to study in general how the variance of the reweighted observable is affected by the fluctuations of the weights. Given a generic weight function $w$ and observable $O$

$$
\langle O\rangle=\frac{\langle O w\rangle_{w}}{\langle w\rangle_{w}}, \quad \text { with } \quad\langle O\rangle_{w}=\frac{\int d U w^{-1} e^{-S} O}{\int d U w^{-1} e^{-S}},
$$

the variance of the reweighted observable depends on $w$ according to the following equation

$$
\operatorname{var}_{w}(O)=\left\langle w^{-1}\right\rangle\left\langle(O-\bar{O})^{2} w\right\rangle .
$$

In Figure 3 (right panel) we plot eq. (5.4) with $O$ being the energy density measured at flow time $t=t_{0}$ and $W$ the reweighting factor as a function of $\mu$. As a gluonic observable its fluctuations are expected to be little correlated to those of the reweighting factor. Hence $\operatorname{var}_{w}\left(E\left(t_{0}\right)\right)$ is largely independent of the choice of the twisted-mass regulator and this is confirmed by a negligible increase of the variance in Figure 3 with $\mu$. The situation is different for mesonic correlators and needs more investigation.

\section{Conclusions}

In these Proceedings we have discussed the effects induced by the open boundaries on some gluonic and fermionic observables. They are dominated by discretization errors which however decrease rapidly with the lattice spacing. We have shown that vacuum expectation values of scalar quantities, such as $t_{0}$ or the meson masses, can be taken in a safe region in the bulk of the lattice. 
We have analysed different strategies to extract pseudoscalar decay constants (in the presence of boundaries) and we have also proposed a new method. At the moment, none seems to be preferable over the others. The breaking of translation invariance in time given by the boundaries does not introduce a significant disadvantage in terms of precision and spectral quantities can be computed with sub-percent accuracy. In particular source displacement does not reduce the final statistical error.

We have studied the effects of a twisted-mass regulator in the light sector. Also with the reweighting, we are able to obtain results with a small statistical uncertainty. The total cost or benefit of this method is difficult to assess: a cheaper simulation on the one hand has to be confronted with a (slightly) increased uncertainty on the other. For now, we conclude that the method works well.

\section{Acknowledgments}

We thank B. Leder for collaboration in an early stage of the work. We would also like to thank D. Djukanovic, G. P. Engel, A. Francis, G. Herdoiza, H. Horch, M. Papinutto, E. E. Scholz, J. Simeth, H. Simma and W. Söldner for their effort in the production of the ensembles and for many useful discussions. We acknowledge PRACE for awarding us access to resource FERMI based in Italy at CINECA, Bologna and to resource SuperMUC based in Germany at LRZ, Munich. We had access to HPC resources in the form of a regular GCS/NIC project ${ }^{3}$, a JUROPA/NIC project ${ }^{3}$ and through PRACE-2IP, receiving funding from the European Community's Seventh Framework Programme (FP7/2007-2013) under grant agreement RI-283493. This work is supported in part by the grants SFB/TR9 of the Deutsche Forschungsgemeinschaft.

\section{References}

[1] M. Lüscher and S. Schaefer, JHEP 1107 (2011) 036, [arXiv: 1105.4749 ].

[2] M. Lüscher and F. Palombi, PoS LATTICE2008 (2008) 049, [arXiv: 0810 . 0946 ].

[3] P. Korcyl, PoS LATTICE2014 (2014) 029.

[4] M. Bruno, D. Djukanovic, G. P. Engel, A. Francis, G. Herdoiza, et al. arXiv: 1411.3982.

[5] M. Lüscher, JHEP 1008 (2010) 071, [arXiv: 1006 . 4518].

[6] ALPHA Collaboration, M. Guagnelli, J. Heitger, R. Sommer, and H. Wittig, Nucl.Phys. B560 (1999) 465-481, [hep-lat/9903040].

[7] M. Lüscher and S. Schaefer, Comput.Phys.Commun. 184 (2013) 519-528, [arXiv:1206.2809].

[8] A. Hasenfratz and A. Alexandru, Phys.Rev. D65 (2002) 114506, [hep-lat/ 0203026 ].

[9] M. Hasenbusch, Phys.Lett. B519 (2001) 177-182, [hep-lat/ 0107019 ].

[10] A. Hasenfratz, R. Hoffmann, and S. Schaefer, Phys.Rev. D78 (2008) 014515, [arXiv : 0805.2369 ].

\footnotetext{
${ }^{3}$ http://www.fz-juelich.de/ias/jsc/EN/Expertise/Supercomputers/ComputingTime/Acknowledgements.html
} 